\title{
Fabrication of Nanostructured Objects by Thermal Vacuum Deposition of Ge Films onto (100)GaAs Substrates
}

\author{
Vitalii Borblik*, Andrey Korchevoi, Andrii Nikolenko, Viktor Strelchuk, Alexander Fonkich, \\ Yurii Shwarts, Marina Shwarts
}

Institute of Semiconductor Physics, National Academy of Sciences of Ukraine, Kiev, Ukraine

Copyright $(2016$ by authors, all rights reserved. Authors agree that this article remains permanently open access under the terms of the Creative Commons Attribution License 4.0 International License

\begin{abstract}
The technique of thermal vacuum deposition of Ge onto GaAs substrates has been used for obtaining nanocrystalline Ge films. Nanocrystalline character of the films is confirmed by atomic force microscopy of their surface and by the data of Raman light scattering. The most probable size of the nanocrystallites forming the films decreases monotonically with decreasing their thickness. And raise of the deposition temperature results in their enlargement. Electro conductivity of such films proves to be high enough (resistivity of $1-10 \mathrm{Ohm} \mathrm{cm}$ at room temperature) and has character of variable range hopping conduction of the Mott's type. The hops, presumably, take place through the localized states connected with the grain boundaries.
\end{abstract}

Keywords Thermal Vacuum Deposition, Germanium, Gallium Arsenide, Nanocrystalline Films, Hopping Conduction

\section{Introduction}

Thermal vacuum deposition of Ge films onto GaAs substrates were studied widely in the sixties (see, for example, [1]) for the purpose of use them in electronics. But because of unsatisfactory quality of such films for device applications (defectiveness, amorphous or polycrystalline character), simple method of evaporation has been forced out by more perfect technologies (molecular beam epitaxy, chemical-vapor deposition, high-vacuum magnetron sputtering and so on) which has allowed to bring the Ge films on GaAs up to device quality (high carrier mobility, large lifetimes of non-equilibrium carriers etc) [2].

At the same time, a new field of science has appeared, in the framework of which polycrystalline (more exactly nanocrystalline) character of the Ge film is right desirable property. This field of science is nanophysics. Wide investigation of nanocrystalline (or nanostructured) objects is connected with significant changes of all properties of the materials (mechanical, electrical, optical) in nanostructured state [3]. Keeping it in mind we want to return to simple and cheap technology of thermal vacuum deposition.

Nanostructured state of Ge film is attractive, uppermost, in connection with the possibility to obtain a luminescence in visible range of the spectrum. It is important also that these films should have high enough electro conduction that would allow obtaining active light emitting devices thereupon.

In this connection, we report here about using this simple and low-cost method for deposition of Ge onto substrates of (100)GaAs and (111)Si. Different regimes of the film deposition have been studied and those of them which result in nanocrystalline Ge films have been found. Then structural (by means of Raman spectroscopy and atomic force microscopy) and electric properties of such films have been investigated.

\section{Experimental}

The crucible made of tantalum-graphite alloy which was heated by alternate current $(\sim 40-50 \mathrm{~A})$ up to temperature of $\sim 1400-1600^{\circ} \mathrm{C}$ and produced thermal evaporation of $\mathrm{Ge}$, was located in vacuum camera with pressure of $(2-5) \cdot 10^{-6}$ Torr. Temperature of the (100) GaAs substrate with resistivity of $10^{8} \mathrm{Ohm} \mathrm{cm}$ could be supported in the range of $200-550^{\circ} \mathrm{C}$ by the special heater made of molybdenum. The same heater was used for the substrates annealing allowing temperature up to $1100^{\circ} \mathrm{C}$. Chromel-alumel thermocouples were used for temperature control with accuracy of $\pm 5^{\circ} \mathrm{C}$. For control of starting and finishing of the deposition process, a mechanical shutter made of tantalum was located between the evaporator and the substrate.

The film thickness was measured by means of micro profilometer "Alpha-Step 100" with accuracy of $4 \mathrm{~nm}$ and by micro interferometer MII-4. Technological characteristics of the obtained samples are presented in Table 1. 
Table 1. Technological characteristics of the samples

\begin{tabular}{|c|c|c|c|c|c|c|}
\hline \multirow[b]{2}{*}{ Number } & \multirow[b]{2}{*}{ Film-substrate } & \multicolumn{2}{|c|}{ Substrate annealing } & \multicolumn{3}{|c|}{ Conditions of the deposition } \\
\hline & & $\begin{array}{c}\text { Annealing } \\
\text { temperature, }{ }^{0} \mathrm{C}\end{array}$ & $\begin{array}{c}\text { Annealing } \\
\text { duration, min }\end{array}$ & $\begin{array}{c}\text { Substrate } \\
\text { temperature, }{ }^{0} \mathrm{C}\end{array}$ & $\begin{array}{c}\text { Deposition duration, } \\
\text { sec } \\
\end{array}$ & $\begin{array}{c}\text { Film } \\
\text { thickness, nm } \\
\end{array}$ \\
\hline 2 & Ge-GaAs & 500 & 20 & 500 & 70 & 150 \\
\hline 3 & Ge-GaAs & 500 & 20 & 350 & 70 & 150 \\
\hline 8 & Ge-GaAs & 400 & 5 & 400 & 70 & 150 \\
\hline 12 & Ge-GaAs & 400 & 8 & 400 & 35 & 75 \\
\hline 15 & Ge-GaAs & 430 & 5 & 450 & 70 & 150 \\
\hline 16 & Ge-GaAs & 400 & 6 & 380 & 70 & 150 \\
\hline 20 & Ge-GaAs & 400 & 8 & 380 & 70 & 150 \\
\hline 22 & Ge-GaAs & 400 & 8 & 380 & 210 & 450 \\
\hline 23 & Ge-GaAs & - & - & 400 & 7 & 15 \\
\hline
\end{tabular}

Measurements of the micro Raman light scattering were carried out in reflection geometry at room temperature by means of triple Raman spectrometer T-64000 Horiba Jobin-Yvon equipped with cooled CCD detector. For the samples excitation, light from Ar-Kr ionic laser with wavelength of $488 \mathrm{~nm}$ are focused on the sample by objective $50 \mathrm{x} / 0.75$ in a spot of $\sim 1 \mu \mathrm{m}$ size with the radiation power near $1 \mathrm{~mW}$.

Investigations of the film surface were carried out by atomic force microscope Nano Scope IIIa Dimension 3000 (Digital Instruments, USA) in the regime of periodic contact. The serial silicon probes NSG-11 with nominal tip curvature radius of $10 \mathrm{~nm}$ (NT-MDT, Russia) were used.

For measuring conductive properties of the films, planar contacts were deposited with spacing of $1 \mathrm{~mm}$ between them.

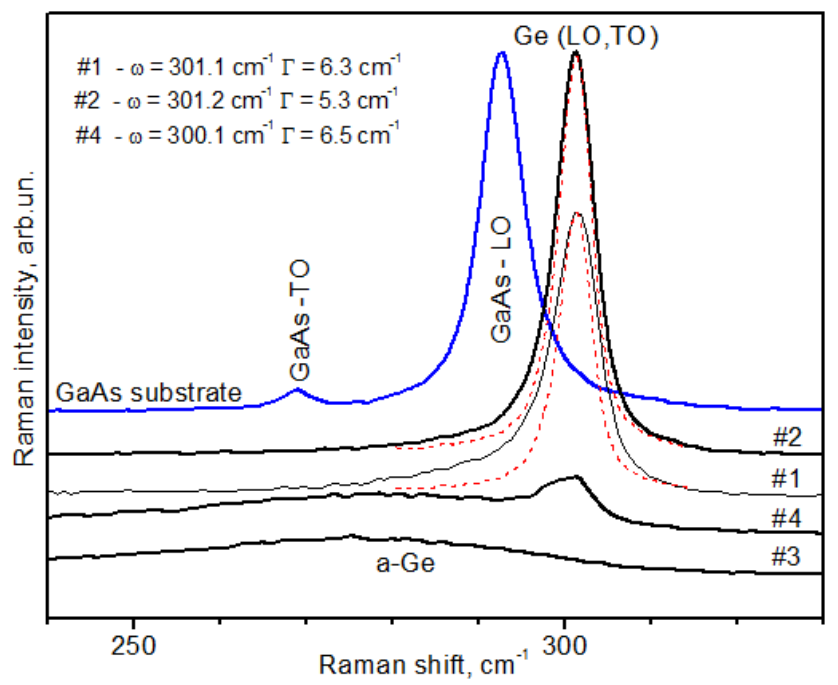

Figure 1. Spectra of the Raman light scattering for samples \#1-4; dashed curves represent the spectrum of bulk Ge.

\section{Results and Discussion}

\subsection{Technological Conditions for Nanocrystallinity of the Ge Films}

Figure 1 represents the data of Raman light scattering for 4 films: $\# 2$ and $\# 3$ - deposited at temperature of $500^{\circ} \mathrm{C}$ and $350^{\circ} \mathrm{C}$, respectively, on the GaAs substrates annealed at $500^{\circ} \mathrm{C}$ for $20 \mathrm{~min}, \# 4$ - deposited at temperature of $300^{\circ} \mathrm{C}$ on non-annealed GaAs substrate and \#1 - deposited at the same temperature on non-annealed Si substrate.

At low deposition temperature $\left(350^{\circ} \mathrm{C}\right)$, Ge film turns out to be amorphous as it follows from style of the curve for sample \#3 in the form of diffuse peak. But at deposition temperature of $500^{\circ} \mathrm{C}$ (sample \#2) the sharp peak appears near the optic phonon frequency of $300 \mathrm{~cm}^{-1}$ which corresponds to crystalline Ge. However this peak is broadened in comparison with that in the bulk Ge (dashed curve in Figure 1) due to polycrystalline (grain) structure of the obtained film and it is asymmetrical due to effect of phonons quantum confinement in the nano-dimensional crystallites. The film deposited at $300^{\circ} \mathrm{C}$ on non-annealed GaAs substrate (sample \#4) turns out to be amorphous as well (though with some portion of crystalline phase) and the film deposited at the same temperature on non-annealed $\mathrm{Si}$ substrate (sample \#1) proves to be polycrystalline (with rather large broadening and asymmetry).

\subsection{Structure of the Films Surface}

Results of the atomic force microscopy (AFM) are represented in Figure 2 for a number of the films (obtained by $\mathrm{Ge}$ deposition at temperature of $380^{\circ} \mathrm{C}$ onto the GaAs substrates annealed previously at $400^{\circ} \mathrm{C}$ for $8 \mathrm{~min}$ ) which have different thicknesses $-450,150$ and $75 \mathrm{~nm}$. Figure 3 represents analogous data for even more thin film with thickness of $15 \mathrm{~nm}$ obtained under somewhat different conditions - deposition at $400^{\circ} \mathrm{C}$ onto non-annealed GaAs substrate. 

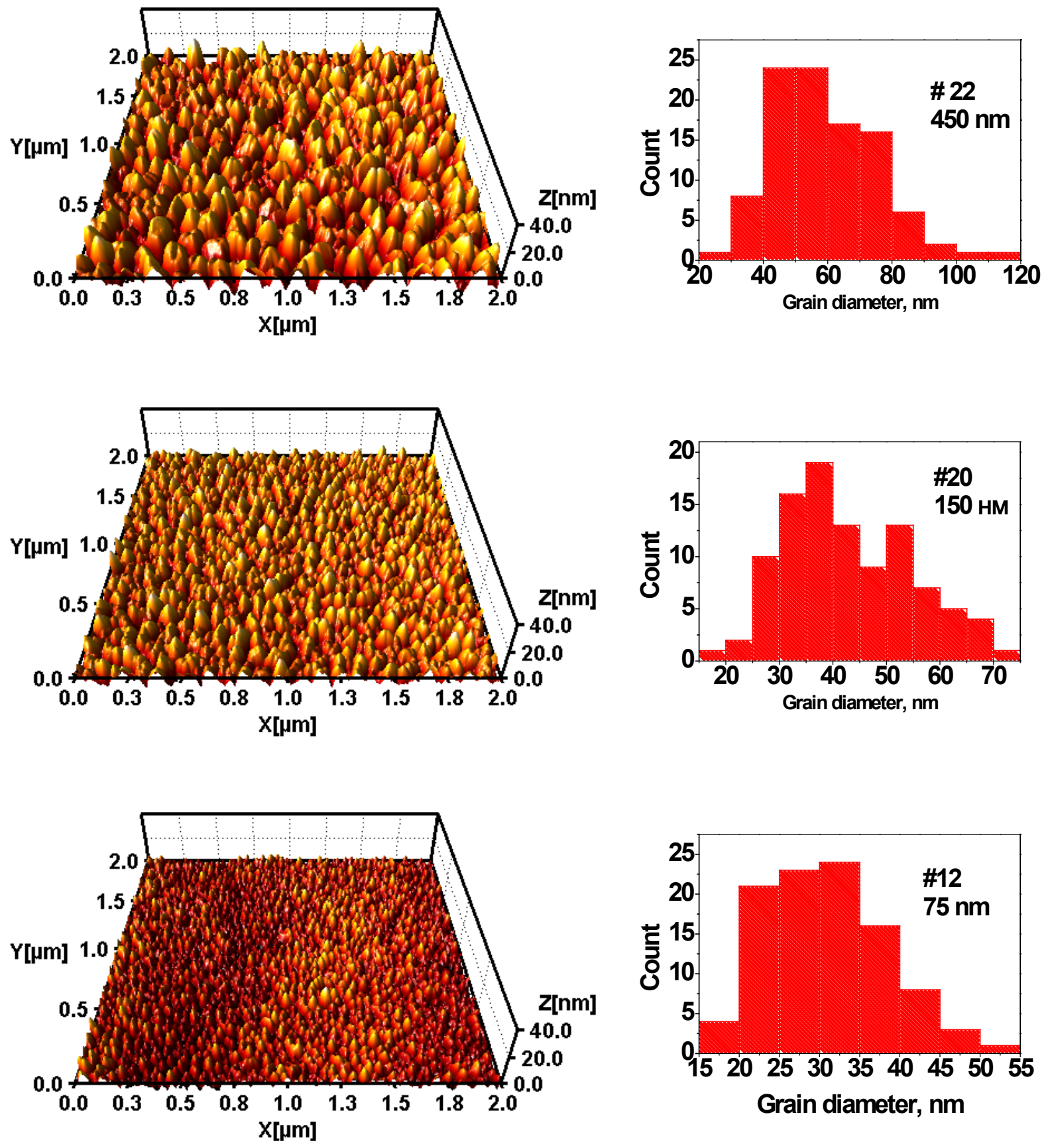

Figure 2. Images of the film surface in atomic force microscope and histograms of the lateral grain sizes for Ge films with the thickness of 450 (a), 150 (b) и $75 \mathrm{~nm}$ (c) on GaAs substrates annealed at $400^{\circ} \mathrm{C}$ for $8 \mathrm{~min}$. 

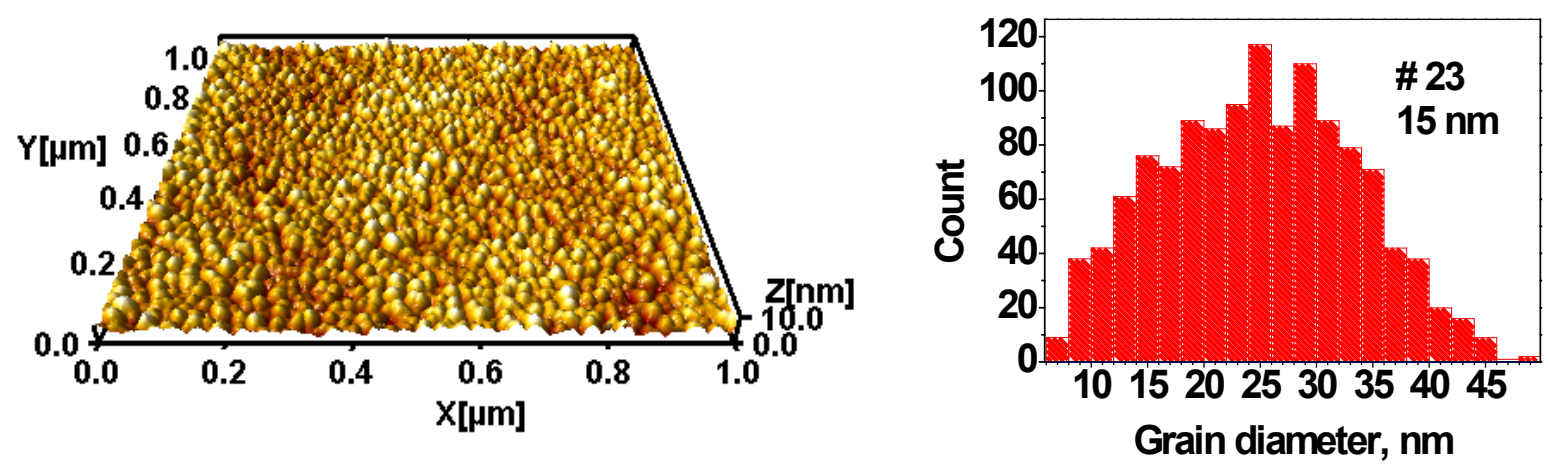

Figure 3. The same as in Figure 2 for Ge film with thickness of $15 \mathrm{~nm}$ on non-annealed GaAs substrate.

All of them have a grain, polycrystalline, structure. But characteristic grain sizes turn out to be different depending on the film thickness. Distributions of the effective lateral grain sizes are presented in corresponding histograms in Figures 2 and 3 , and distributions of the vertical scales of the surface relief are presented in Figure 4 (along with the results of their processing as the gauss curves).

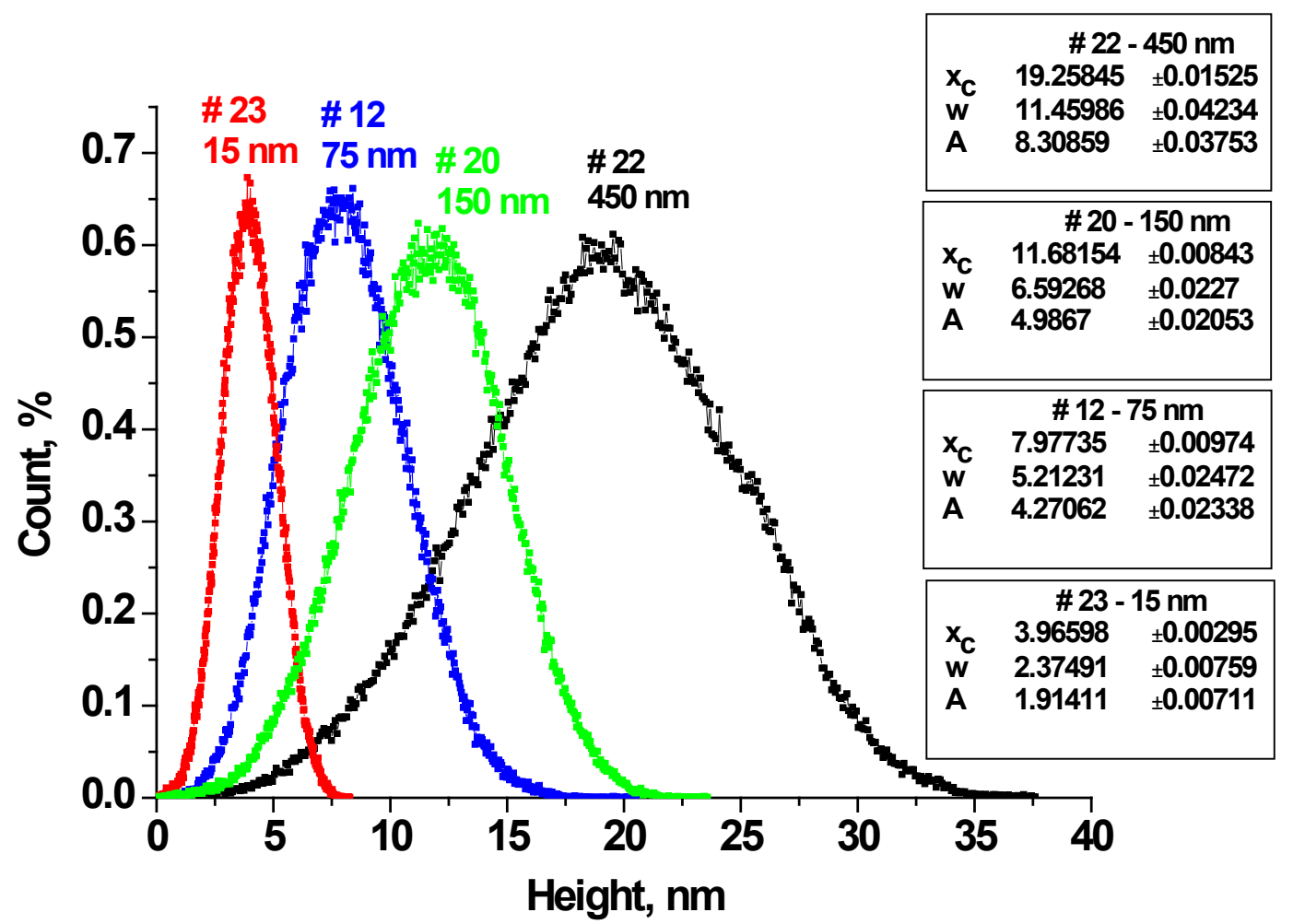

Figure 4. Distributions of the height probabilities in surface relief of the films shown in Figures 2 and 3 along with the results of their approximation by gauss curves (by means of program Origin); here $x_{c}$ is coordinate of the curve maximum (the most probable value), $w / 2$ is standard deviation from the distribution center (roughness), $A$ is area under the curve. 
As it follows from the data represented, decrease in the film thickness (from $450 \mathrm{~nm}$ to $15 \mathrm{~nm}$ ) results in decreasing all characteristic scales of their surface relief. So, the most probable lateral grain size decreases from 40-60 nm through $35-40 \mathrm{~nm}$ (then $30-35 \mathrm{~nm}$ ) down to $24-26 \mathrm{~nm}$. The most probable value of the relief height decreases from $19.26 \mathrm{~nm}$ through $11.68 \mathrm{~nm}$ (then $7.98 \mathrm{~nm}$ ) down to $3.97 \mathrm{~nm}$, and the root-mean-square height deviation from the average value (i.e. roughness) - from $5.73 \mathrm{~nm}$ through $3.3 \mathrm{~nm}$ (then 2.60 $\mathrm{nm}$ ) down to $1.18 \mathrm{~nm}$. It is obvious that enlargement of the grain size with increase in the film thickness is connected directly with increase in the time spent for their growth.

It is interesting that qualitative similarity dependence between film thickness and grain size has been observed in the case of hydrogenised germanium films on glass [4]. As was noted in [4], the values obtained by means of AFM prove to be somewhat overrated because of the finite tip radius; therefore the lateral scale of the film nanostructure may turn out even smaller actually.

Above obtained dependencies of the characteristic scales of the surface relief on the film thickness are summarized in Figure 5. It is worthwhile to pay attention to practically complete identity of the thickness dependencies of the lower boundary of characteristic scales for lateral grain sizes (a, bottom) and of their characteristic height (b).

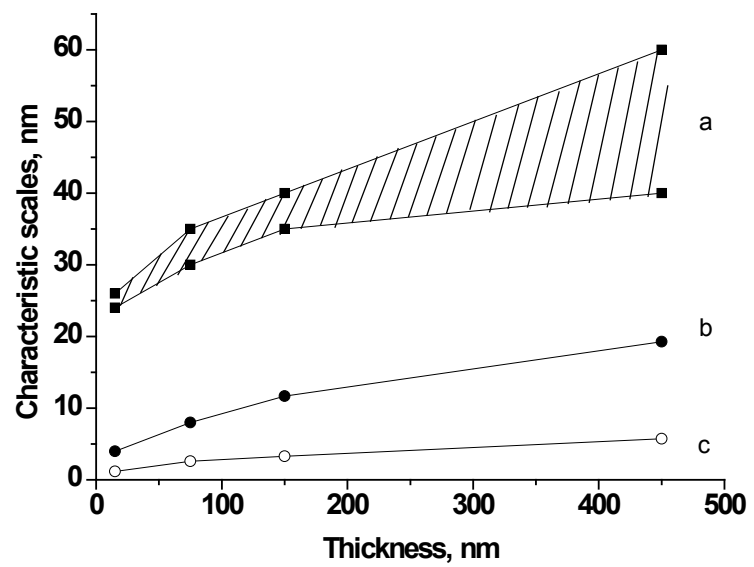

Figure 5. Dependences of the most probable characteristic lateral size of the nanocristallites (a), the characteristic scale of the relief height (b) and the root-mean-square deviation of the height from its average value (roughness) (c) on the film thickness.

In all the cases, a ratio of the double characteristic height to the characteristic lateral size is less than 1, i.e. the grains (at least, in the near-surface layer) are oblate in direction perpendicular to the surface, and their oblateness increases with decrease in the film thickness. In Figure 6 this fact is demonstrated for the thinnest film $(15 \mathrm{~nm})$ where the height of each element of the film surface relief is plotted versus its effective lateral size. It should be noted that an oblateness of the grains has been observed also on the surface of films consisting of Ge nanocrystals embedded in $\mathrm{SiO}_{2}$ matrix [5] and during self-assembled growth of Ge quantum dots on strained SiGe sublayers [6].

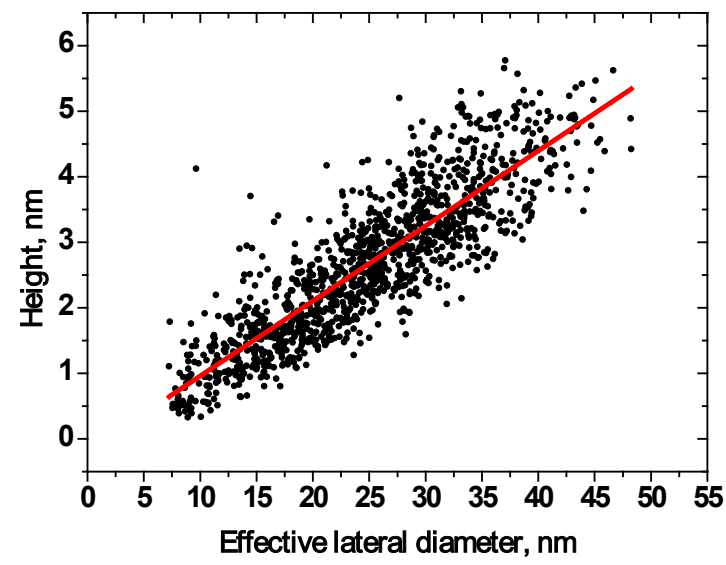

Figure 6. Relationship between height of the surface relief element and its effective lateral size in the film with thickness of $15 \mathrm{~nm}$

\subsection{Subsurface Study of Polycrystalline Character of the Same Samples}

The statement that above characteristic scales of the film surface relief characterize just the nanocrystallites sizes (not scales of the surface inhomogeneity) is proved by the results of Raman spectroscopy for the same samples because the probing light penetrates into the films deeply. Corresponding data are presented in Figure 7. It is seen that as the film thickness decreases and the nanocrystallites become less, the Raman curve for optic phonon of Ge becomes more and more asymmetrically widened and its maximum shifts to lower energies. This is a sequence of the quantum confinement effect in optic phonons. The additional peak which appears in the more thin films corresponds to frequency of the longitudinal optic phonon of GaAs substrate whereto the probing light more and more penetrates.

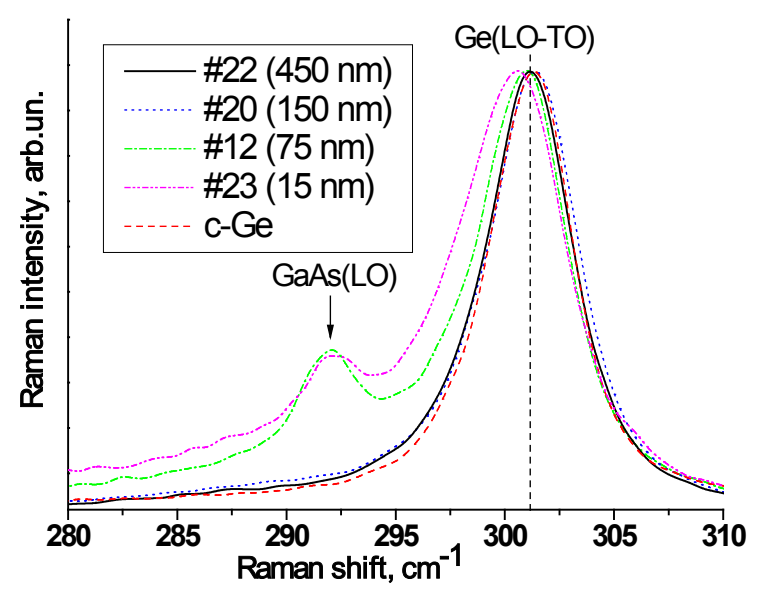

Figure 7. Spectra of Raman light scattering for samples \#12,\#20,\#22, and \#23; dashed curve represents spectrum of bulk Ge.

\subsection{Effect of Deposition Temperature on Nanocrystallites Size}

The substrate temperature under deposition process also 
influences on size of the nanocrystallites. Let us trace this for GaAs substrate under other similar conditions: these are rather like previous substrate annealing at $400^{\circ} \mathrm{C}$ for 5-6 $\mathrm{min}$ and the same thickness of the Ge films $(150 \mathrm{~nm})$.

Figure 8 presents AFM images of the films surface and their characteristic lateral scales at three deposition temperatures $-380^{\circ} \mathrm{C}, 400^{\circ} \mathrm{C}$ and $450^{\circ} \mathrm{C}$ (samples \#16, \#8 and \#15, respectively) and Figure 9 shows corresponding probabilities distribution for their relief heights along with results their approximation by the gauss curves.
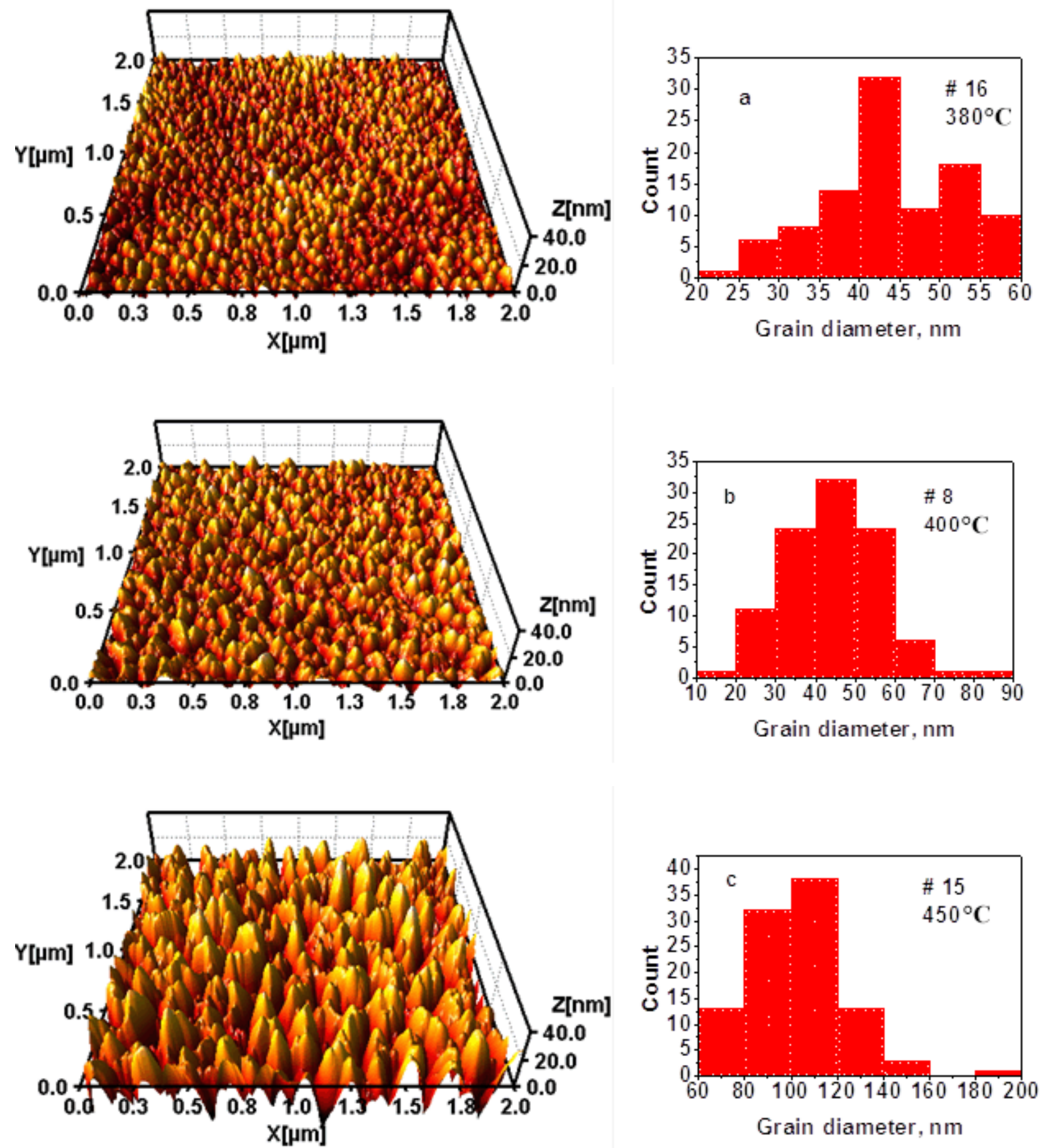

Figure 8. AFM images of the film surfaces and histograms of the lateral grain sizes for the Ge films with thickness of $150 \mathrm{~nm}$ at the deposition temperature of $380^{\circ} \mathrm{C}(\mathrm{a}), 400^{\circ} \mathrm{C}(\mathrm{b})$ and $450^{\circ} \mathrm{C}(\mathrm{c})$. 


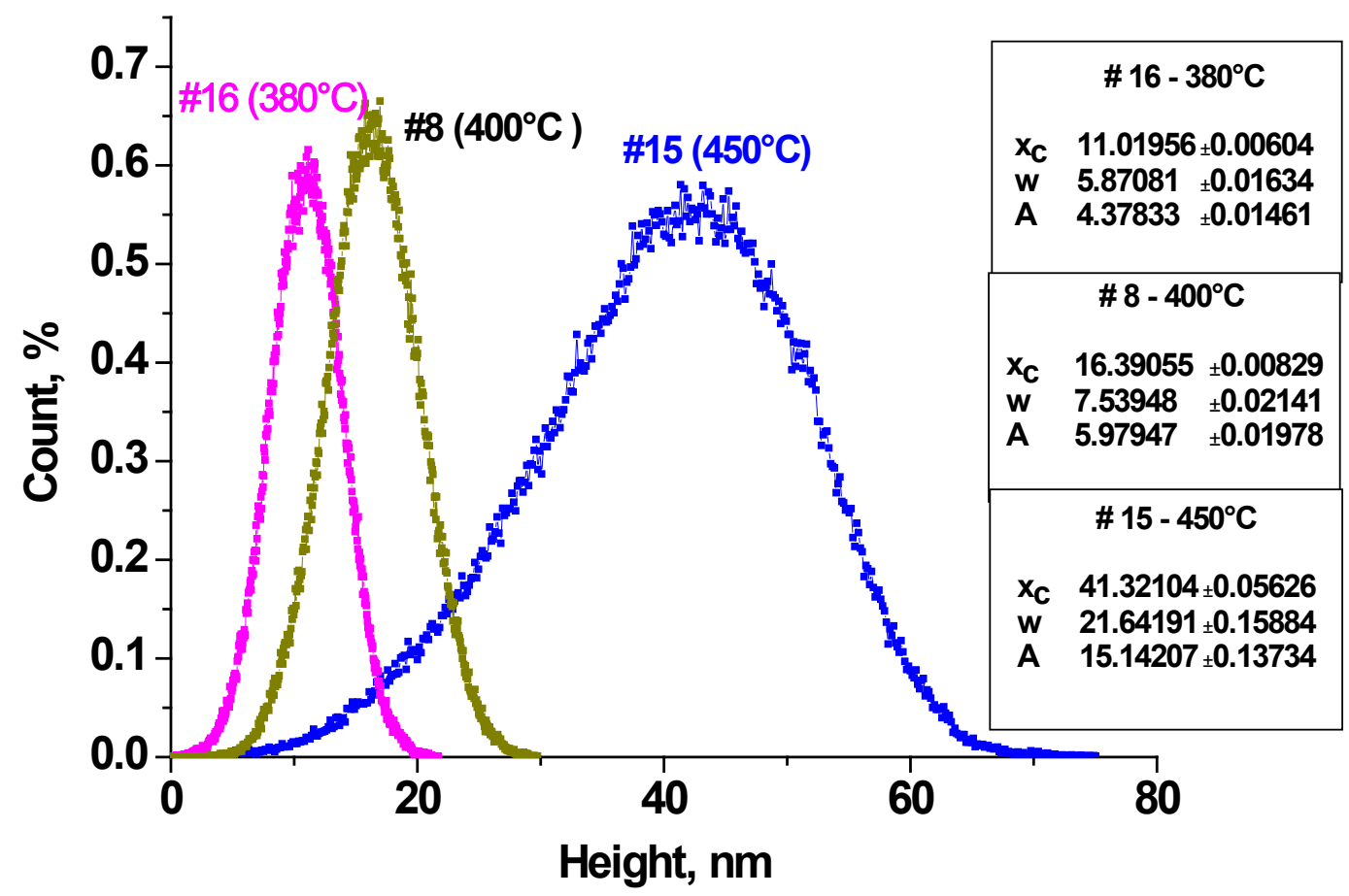

Figure 9. Distribution of the height probabilities for surface relief of the same films as in figure 8 along with results their approximation by the gauss curves.

It is obvious that all characteristic scales of the surface relief increase with growth of the deposition temperature: from $40-45 \mathrm{~nm}$ through $40-50 \mathrm{~nm}$ up to $100-120 \mathrm{~nm}$ for lateral sizes and from $11.02 \mathrm{~nm}$ through $16.38 \mathrm{~nm}$ up to $41.32 \mathrm{~nm}$ for vertical ones. It should be underlined that the deposition times were equal. Ratio of double characteristic height of the surface relief to characteristic lateral scale (that characterizes the oblateness) firstly increases with the deposition temperature and then saturates being always less than 1 .

\subsection{Conductive Properties of the Films}

All obtained films had linear current-voltage characteristics up to applied voltage of $10 \mathrm{~V}$ in temperature range of 77-300 K. The measurements were carried out in longitudinal direction, i.e. along the films.

Figure 10 represents Arrhenius curve for the current $I$ at the fixed voltage $U=1 \mathrm{~V}$ for sample \#16 (the film with thickness of $150 \mathrm{~nm}$ deposited at temperature of $380^{\circ} \mathrm{C}$ onto GaAs substrate annealed at $400^{\circ} \mathrm{C}$ for $6 \mathrm{~min}$ ). This curve determines temperature dependence of the film electro conductivity $\sigma$ which (as it is seen from figure) is characterized by variable activation energy. The latter may be defined here as a derivative $-d \ln I / d(k T)^{-1}$.

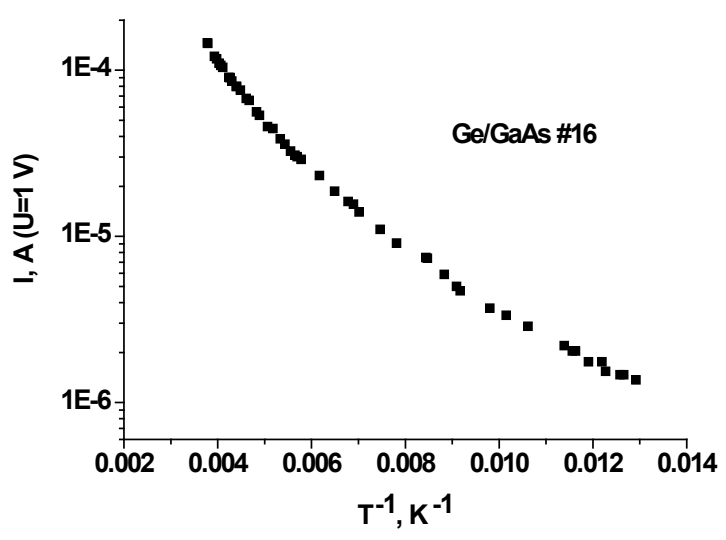

Figure 10. Arrhenius curve for the current along the film at the fixed voltage of $1 \mathrm{~V}$ for sample \#16.

This dependence is straightened well being plotted as a function of $(1 / T)^{1 / 4}$ as it is demonstrated by Figure 11. It follows from this that the film conductivity is realized by means of variable range hopping conduction of Mott's type when $\ln \sigma \propto\left(T_{0} / T\right)^{1 / 4}$. Here $T_{0}=$ const $/ \mathrm{kg}\left(E_{F}\right) a^{3}$ where $k$ is the Boltzmann constant, $a$ is localization radius of the states through which the hops take place, $g\left(E_{F}\right)$ is density of these states on the Fermi level. 


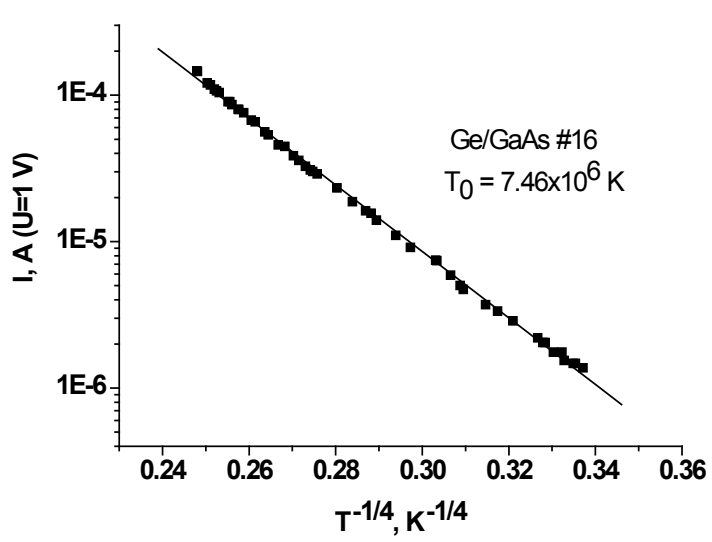

Figure 11. Temperature dependence of the current along the film at the fixed voltage of $1 \mathrm{~V}$ for sample $\# 16$ as a function of $(1 / T)^{1 / 4}$.

The same type of conductivity (hopping one, with the variable activation energy) is known also in another nanostructured objects - for example, in the films containing Ge nanoclusters in $\mathrm{SiO}_{2}$ matrix [7] (in [7], conductivity was measured across the films). Authors of this paper have observed decrease in $T_{0}$ with growth of the Ge content in the films, i.e. with increase in either sizes or number of the conductive clusters (that results in increasing $g\left(E_{F}\right)$ ).

Note that in the case when $\mathrm{Ge}$ in $\mathrm{SiO}_{2}$ matrix has been transformed into nanocrystalline inclusions with sizes of 4-9 $\mathrm{nm}[8]$ (as a result of following thermo-treatment), the same authors have observed the temperature dependence of the film conductivity in the form of $\ln \sigma \propto\left(T_{0} / T\right)^{1 / 2}$. In spite of similarity of this dependence with that for variable range hopping conduction under condition of coulomb gap [9], this mechanism is not considered as appropriate to given situation.

The temperature dependence in the form of $\ln \sigma \propto\left(T_{0} / T\right)^{1 / 4}$ has been observed also in paper [10] for the conductivity along the films of similar type (Ge nanoparticles embedded in $\mathrm{SiO}_{2}$ matrix) and for the conductivity across the films which consist of $\mathrm{Ge}$ nanocrystals on silicon or silica substrates grown at room temperature [11]. In all similar cases, the variable range hopping conduction of Mott's type is explained by existence of the localized states connected with the grain boundaries.

\section{Conclusions}

Thus

- condition of obtaining nanocrystalline structure of Ge films on GaAs substrate is the high enough substrate temperature under deposition (not lower than $380-400^{\circ} \mathrm{C}$ ),

- the thinner the film, the smaller are the crystallites forming it; this allows us to choose purposefully their desirable size (at the average),

- typical size of the nanocrystallites in the film depends also on deposition temperature (increases with its growth),
- lateral size of the nanocrystallites proves to be larger than their vertical size i.e. nanocrystallites have oblate form in direction perpendicular to the surface;

- electro conductivity of such films has a character of variable range hopping conduction of Mott's type i.e. nanostructured Ge films may be considered as one more variant of granular media which are studied widely at present,

- in view of smallness of the distances between the adjacent nanograins in the films, their conductivity proves to be high enough (resistivity of $1-10 \mathrm{Ohm} \mathrm{cm}$ at room temperature) that may allow creation of active light emitting devices on the base of such films.

\section{Acknowledgements}

This work has been supported by the National Academy of Sciences of Ukraine (project 2.2.6.34).

\section{REFERENCES}

[1] R. F. Lever, E. J. Huminski. Epitaxy of germanium films on gallium arsenide by vacuum evaporation, J. Appl. Phys., Vol.37, No. , 3638-3639, 1966.

[2] S.-H. Tang, E. Y. Chang, M. Hudait, J.-S. Maa, C.-W. Liu, G.-L. Luo, H.-D. Trinh, Y.-H. Su. High quality Ge thin film grown by ultrahigh vacuum chemical vapor deposition on GaAs substrate, Appl. Phys. Lett. Vol.98, No.16, 161905(1-3), 2011.

[3] H. Gleiter. Nanostructured materials: basic concepts and microstructure, Acta Materialia, Vol.48, No.1, 1-29, 2000.

[4] W. B. Jordan, E. D. Carlson, T. R. Johnson, S. Wagner. Structural evolution of nanocrystalline germanium thin films with film thickness and substrate temperature, Mat. Res. Soc. Symp. Proc. Vol.762, paper A6.5.1, 2003.

[5] I. Stavarache, A.-M. Lepadatu, T. Stoica, M. L. Ciurea. Annealing temperature effect on structure and electrical properties of films formed of Ge nanoparticles in $\mathrm{SiO}_{2}$, Appl. Surf. Sci., Vol.285( Part B), 175-179, 2013.

[6] M. Ya. Valakh, P. M. Lytvyn, A. S. Nikolenko, V. V. Strelchuk, Z. F. Krasilnik, D. N. Lobanov, A. V. Novikov. Gigantic uphill diffusion during self-assembled growth of Ge quantum dots on strained SiGe sublayers, Appl. Phys. Lett., Vol.96, No.14, 141909, 2010.

[7] M. Fujii, Y. Inoue, S. Hayashi, K. Yamamoto. Hopping conduction in $\mathrm{SiO}_{2}$ films containing $\mathrm{C}, \mathrm{Si}$, and Ge clusters, Appl. Phys. Lett., Vol.68, No.26, 3749-3751, 1996.

[8] M. Fujii, O. Mamezaki, S. Hayashi, K. Yamamoto. Current transport properties of $\mathrm{SiO}_{2}$ films containing Ge nanocrystals, J. Appl. Phys., Vol.83, No.3, 1507-1512, 1998.

[9] B. I. Shklovskii, A. L. Efros. Electron Properties of Doped Semiconductors, Springer, Berlin, 1984.

[10] I. Stavarache, A.-M. Lepadatu, A. V. Maraloiu, V. S. 
Teodorescu, M. L. Ciurea. Structure and electrical transport in films of Ge nanoparticles embedded in $\mathrm{SiO}_{2}$ matrix, J. Nanopart. Res., Vol.14, No.7, Article: 930(1-9), 2012.
[11] S. Banerjee. Non-ohmic hopping conduction in Ge nanocrystalline film, Physical E, Vol.15, No.3, 164-168, 2002. 